\title{
Landfill Gas Monitoring Network Development of Wireless Sensor Network Platforms
}

\author{
Fiachra Collins ${ }^{1}$, Dylan Orpen ${ }^{1}$, Eoghan McNamara ${ }^{1}$, Cormac Fay ${ }^{1}$ and Dermot Diamond ${ }^{1}$ \\ ${ }^{1}$ CLARITY Centre for Sensor Web Technologies, National Centre for Sensor Research, Dublin City University, Glasnevin, \\ Dublin 9, Ireland \\ \{fiachra.collins, dermot.diamond\}@dcu.ie
}

Keywords: Environmental monitoring, gas sensing, landfill gas

\begin{abstract}
A wireless sensor network has been developed for the application of landfill gas monitoring, specifically sensing methane, carbon dioxide and extraction pressure. This collaborative work with the Irish Environmental Protection Agency has been motivated by the need to reduce greenhouse gas emissions as well as aiming to improve landfill gas management and utilisation. This paper describes the preliminary findings of an ongoing trial deployment of multiple sensing platforms on an active landfill facility; data has been acquired for nine months to date. The platforms have operated successfully despite adverse on-site conditions, with validity demonstrated by reasonably strong correlation with independent on-site measurements. The increased temporal and spatial resolution provided by distributed sensor platforms is discussed with regard to improving landfill gas management practice.
\end{abstract}

\section{INTRODUCTION}

Wireless sensor networks are gaining increasing attention with regard to environmental monitoring; the extended temporal and spatial resolution provided by distributed sensing nodes enable a wealth of environmental parameters to be measured. The need for such environmental monitoring is driven by international legislation targeting a reduction in greenhouse gas emissions and improving air quality (EU, 2008). Traditionally, such parameters have been measured using fixed single-point sampling equipment. For example, the Irish Environmental Protection Agency (EPA) operates 29 fixed stations around Ireland for the purposes of monitoring ambient air quality (EPA, 2012). The emergence of medium and large scale sensor networks has been evident in recent years; the first urban network for monitoring carbon dioxide was announced in Oakland, USA (Cohen et al., 2012), while air quality sensor networks have been deployed in urban centres in the UK (Envirowatch, 2012). However, while WSNs are moving to the foreground of attention with respect to environmental monitoring, they have yet to proliferate to their full potential. The principle obstacles to this proliferation are price-point and technology dependability. The extrapolation of current sensor and platform technology costs for scaled-up deployments is, for the most part, not economically viable. Furthermore, the deployment of a distributed network of sensors presents a challenge in terms of configuration, maintenance and multiplication of technical issues.

The environmental monitoring discussed in this paper is specifically with regard to landfill waste facilities. Landfill gas is primarily composed of carbon dioxide $\left(\mathrm{CO}_{2}\right)$ and methane $\left(\mathrm{CH}_{4}\right)$ generated from the anaerobic decomposition of biodegradable waste. Modern landfill facilities contain a network of pipes connecting perforated wells sunk into the waste body, where an applied negative pressure extracts the gases from the waste cells. Landfill gas poses significant environmental hazards, both in terms of greenhouse gas contribution and local hazards such as asphyxiation and combustion. This was dramatically demonstrated in Ireland in early 2011 where a subterranean fire on a closed landfill site incurred local controversy and costly extinguishing and remediation measures.

It can be seen therefore that substantial attention is required to effectively manage landfill gas and indeed utilise it for electricity generation where 
methane concentrations are sufficiently high. From the viewpoint of landfill facility operators, there are two aspects of monitoring landfill gas: firstly at perimeter wells to ensure against gas migration through the waste body into the surrounding soil beyond the confines of the site; secondly in-line with the extraction network to maintain an appropriate gas composition for optimum engine/flare operation. The former aspect of perimeter landfill gas monitoring is what initially motivated this work, resulting in autonomous monitoring platforms being developed and validated (Beirne et al., 2010; Fay et al., 2011; Collins et al., 2012). The latter aspect of in-line monitoring has been the focus of more recent efforts and is the subject of this present paper. Gas management via the site's extraction pipe network is one of the crucial aspects of landfill operation in order to comply with environmental legislation. Given their hazardous nature, all landfill gases must be thermally oxidised (i.e. burned) to mitigate against greenhouse gas emissions and local pollution. For methane concentrations in excess of $50 \% \mathrm{v} / \mathrm{v}$, the gas can be used as fuel for a CHP (combined heat and power) engine, presenting a financial advantage in recuperating costs by generating electricity and selling it to the national grid; otherwise the gas must be burned in a flare. The composition of the gas must be precisely controlled for effective combustion, requiring the adjustment of flow from different waste cells within the landfill site - this operation is called 'field balancing'.

The development of wireless sensor networks is ideally suited to the landfill application, where a typical extraction system covers an expansive area of ground. Moreover, this terrain by nature is difficult to traverse, implying that remote sensors deployed in-situ represent an advantage over the current infrequent manual sampling routines. Gas composition monitoring would indicate the gas generation potential of different waste cells as well as identifying fugitive gas emissions. Measurement of the extraction pressure can be used as a diagnostic tool to identify loss in flow due to blockages or leakages. The distribution of autonomous sensing platforms in a networked configuration would enable more informed and precise field balancing and gas management which, in turn, represents cost savings by promoting electricity generation and avoiding engine/flare downtime.

The paper addresses the research challenge of attaining reliable and accurate sensor performance without incurring prohibitive expense. Typically, cost and performance are inherently linked - cheaper components fail to deliver adequate resolution, accuracy or in-calibration duration. Traditionally, long-term reliability is achievable only at a substantial cost. However, the price of electronics and sensors is continuously reducing, driven by the ever-growing market of consumer technologies and optimised manufacturing techniques. The integration of such technology into deployable platforms with WSN capabilities can fulfil the demand in industry for low-cost long-term sensing. However, market acceptance is only achievable if the sensing performance is validated and proven to work. This is the motivation behind the platform technology described in this paper, which represents a progression of work on developing autonomous gas monitoring platforms with web-based accessibility in collaboration with the Irish EPA.

The rugged construction of these autonomous platforms, as shown in Figure 1(i), enables longterm in-situ monitoring of transient gas events. The system is deployable with GSM communications enabling transmission to a remote base-station, which parses the data onto a database and uploads it onto an online portal. The performance of these platforms has been validated by deployment durations of up to 13 months (Collins, 2011). In
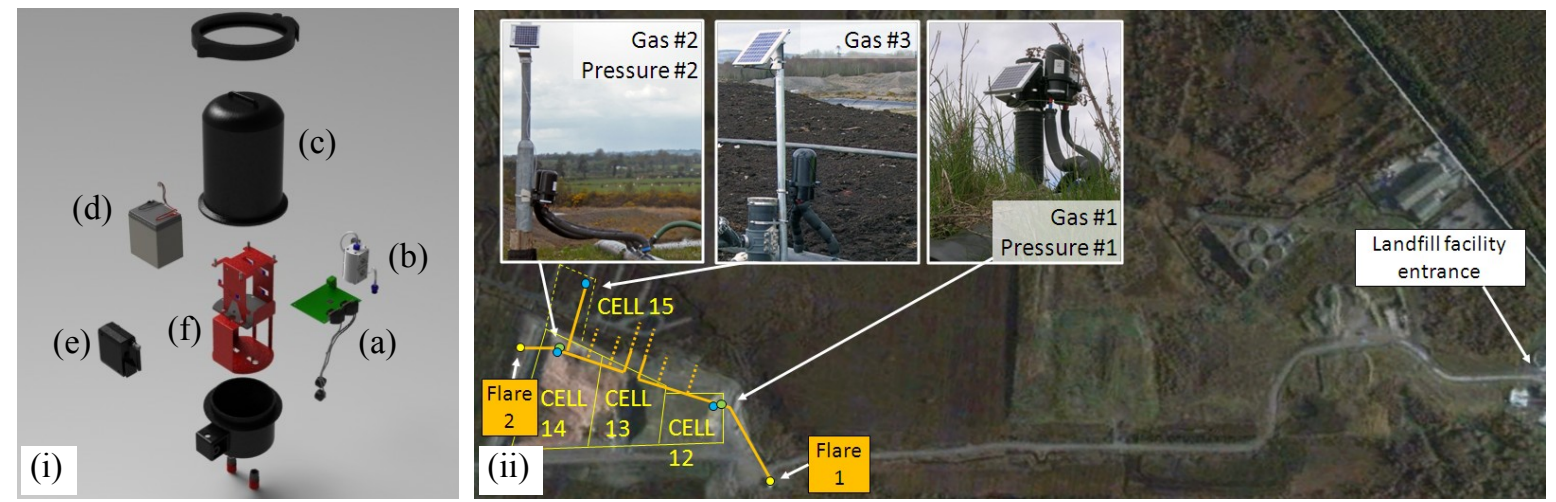

Figure 1: (i) Exploded view of platform (a) microcontroller (MSP430), (b) sensors, (c) IP68 casing, (d) battery, (e) GSM module, (f) extraction pump. (ii) Configuration of wireless sensor network deployed on landfill site. 
addition to the autonomous monitoring operation, analysis of the data provides a value-added service by investigating the factors affecting landfill gas activity, evaluating the contributions of extraction flow-rate and local weather conditions (barometric pressure and rainfall). This paper presents the initialisation of a deployment of multiple sensor platforms on one site, the first of its type on an Irish landfill site.

\section{PLATFORM DEPLOYMENT}

\subsection{Deployment Configuration}

A wireless sensor network in the form of five autonomous platforms has been deployed on a single active landfill site in Ireland. To minimise any debugging issues and allow for flexibility in locating the systems, each of the five systems has been equipped with independent GSM modems for wireless communications. While this may not satisfy the classical definition of a wireless sensor network comprising of a central gateway platform with peripheral node platforms, the five systems nevertheless represents the increased spatial distribution achieved by a sensor network.

There were two configurations of platform deployed on this site: three units were fitted with $\mathrm{CH}_{4}$ and $\mathrm{CO}_{2}$ infrared gas sensors (Premier Series, Dynament, UK) for gas concentration monitoring, two units were fitted with pressure sensors (40PC, Honeywell, UK) for monitoring extraction pressure. The two configurations of platforms employed slightly different data acquisition strategies. The gas platforms ran a sampling routine every 6 hours, with data from that sampling routine being transmitted immediately afterwards. The sample routine consisted of a two-minute sampling period, where the sensors took a reading every three seconds as the pump drew the gas from the landfill's extraction network, resulting in 40 readings each for $\mathrm{CO}_{2}$ and $\mathrm{CH}_{4}$. The two-minute routine enabled a reasonable volume ( $\sim 0.6$ litres) of gas to be sampled, thus avoiding any outliers caused by pockets of concentrated gas. The full dataset of 80 readings was encoded to fit within the 160 character SMS limit, whereupon it was transmitted as a text message to the remote base-station. As the pressure was by nature more variable, readings were acquired every 30 minutes, stored on flash memory and transmitted by SMS every 24 hours. Upon reception in the basestation (based in the lab in DCU), a java program decoded the text message, parsed the data to a database via MySQL and uploaded averaged data to an online portal which could be accessed from a web browser.

The configuration of the deployment is shown in Figure 1(ii). The deployment began in December 2011, with two pairs of gas/pressure platforms deployed in-line with the extraction pipes leading to each of the two flares on site (locations \#1 and \#2 in Figure 1(ii)). The reasoning behind these locations was that the readings could be validated against the flares' SCADA monitoring systems. A third gas monitoring system was deployed in a newly capped cell (location \#3 in Figure 1(ii)) to monitor the gas activity as the waste body matured.

\subsection{On-site Issue Resolution}

As can be expected for outdoor environmental deployments, there were a number of issues to be surmounted including power requirements, wireless communications and sensor readings validity. Furthermore, there were site-specific issues inherent to conditions on the landfill site.

A concern with all remote devices is power provision. For these monitoring platforms, low power 'sleep' algorithms programmed into the microcontroller circuitry enabled a 10-week deployment duration using the $12 \mathrm{~V} 5 \mathrm{Ah}$ lead-acid battery. To avoid system downtime, photovoltaic panels (BP SX-5M) with charge controllers (Solar Technology STS01208) were fitted to the three gas monitoring systems. As seen in Figure 2, solar charging was found to acceptably sustain the battery level of the system indicating an indefinite deployment period in terms of power requirements. Note the $13.5 \mathrm{~V}$ upper limit in Figure 2 is not representative of the actual battery voltage but instead is the maxed-out voltage potential across the solar panel during daylight hours. A truer reading of the battery level is the night-time values - the minima in Figure 2 (ii). Solar charging was not necessary for the pressure monitoring systems which had an estimated battery life of 12 months due to the lack of the pump and power hungry IR sensors.

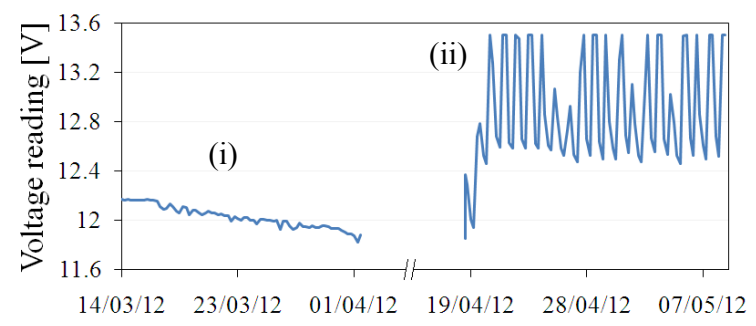

Figure 2: Power levels during operation (i) battery depletion rate, (ii) battery solar recharging 
Setting up communications was straight-forward with acceptable GSM coverage found for the deployment locations on-site. Intra-network communication protocols (RF, etc) were not implemented at this stage due to issues with line-ofsight and the need to simplify the initial network operation. This will be the subject of future development when the scale of the deployments increases. Communications was the principal contributor to the running costs of the platforms; monthly text message costs of $€ 6.30$ and $€ 2.70$ are associated with each gas and pressure system, respectively.

The validity of the sensor readings was confirmed by occasional spot checks using the industry standard equipment (GA2000, Geotech Instruments, UK). Moreover, the gas monitoring platforms were retrieved to the laboratory to test their calibration settings after eight months deployed. As seen in Figure 3, the $\mathrm{CO}_{2}$ sensor was found to have a sensitivity drift of $1.2 \%$ and zero drift of $6.38 \%$; the $\mathrm{CH}_{4}$ sensor had a sensitivity drift of $0.6 \%$ and zero drift of $8.8 \%$. This was considered to be an acceptably small drift considering continual exposure to high gas levels as well as the presence of other noxious and corrosive gases such as hydrogen sulphide $\left(\mathrm{H}_{2} \mathrm{~S}\right)$.

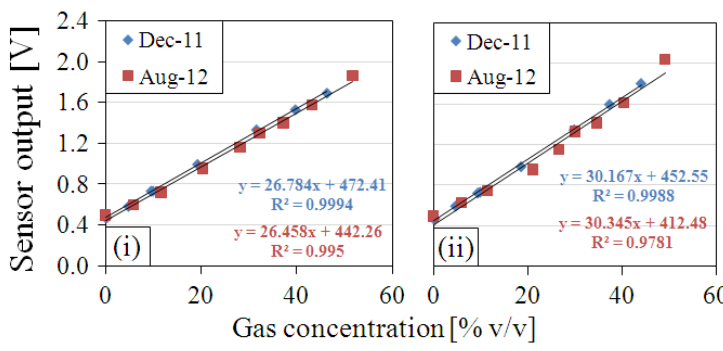

Figure 3: Comparison of sensors' calibration data before and after 8 months deployed (i) $\mathrm{CO}_{2}$, (ii) $\mathrm{CH}_{4}$.

A number of issues specific to this site were encountered and rectified in the early months of the deployment. Firstly, a high moisture content in the landfill's extraction pipes led to condensate forming in the monitoring systems' connection tubing, hence inhibiting the sampling of gas, see Figure 4(i). This was rectified in two ways: the monitoring systems were elevated on their mountings such that any condensate would flow back down into the landfill pipes and the tubing was insulated by neoprene sleeves to reduce the differential temperature between the tubing and the landfill pipes, thus lessening the extent of condensation. The second issue related to the tubing, where an abrupt increase in ambient temperature in April 2012 led to shrinkage of the tubes until disconnection occurred, see Figure 4(ii). The length of the tubing was extended to compensate for this. The third issue concerned the landfill pipe at location \#3; given that this was a newly capped cell, remedial construction works were ongoing to stabilise the pipe which was collapsing from its support ridge due to the weight of excessive water in the pipe, resulting in the breakage of connections on numerous occasions, see Figure 4(iii). These issues were identified from observing the online data, enabling the monitoring operation to be resumed quickly.
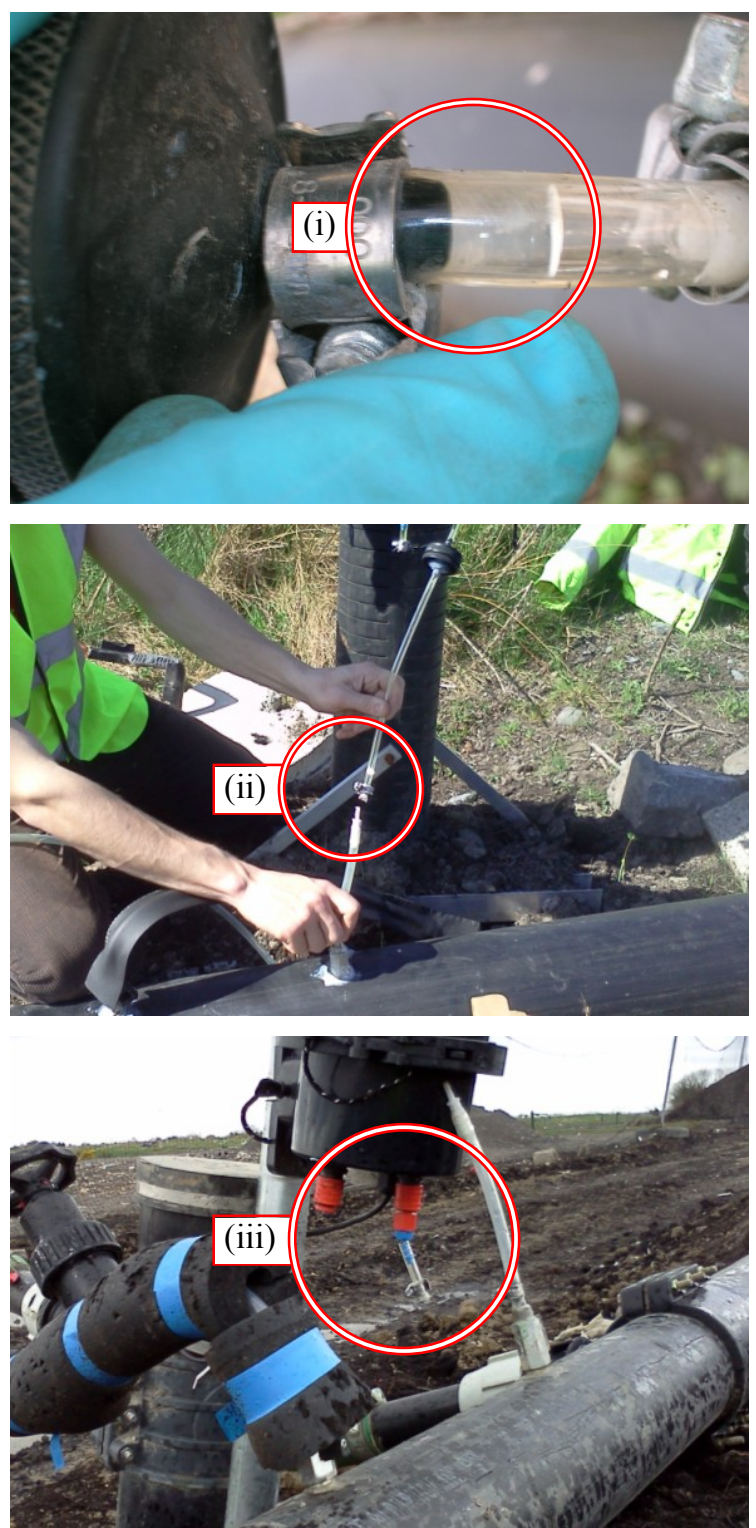

Figure 4: Site-specific issue rectification (i) Waterblockage, (ii) Tube shrinkage, (iii) Connection breakage due to pipe collapse 


\section{RESULTS \& DISCUSSION}

To date, the platforms have operated for up to 9 months, with valid readings totalling over 3,000 and 25,000 for gas concentration and pressure, respectively. Subsets of the data from the sensors in Locations \#1 and \#2 are shown in Figure 5 (some gaps in the data were due to the aforementioned issues described in Section 2). Distinct 'events' are clearly seen in the data, coinciding in both the gas concentration and pressure values. Such evidence of the dynamics of landfill gas concentration has not been available previously; now, for the first time, the cause and effect relationship of landfill gas behaviour can be investigated. Interpretation of this gas field behaviour would be conducive to more informed decisions about the impact of fieldbalancing.
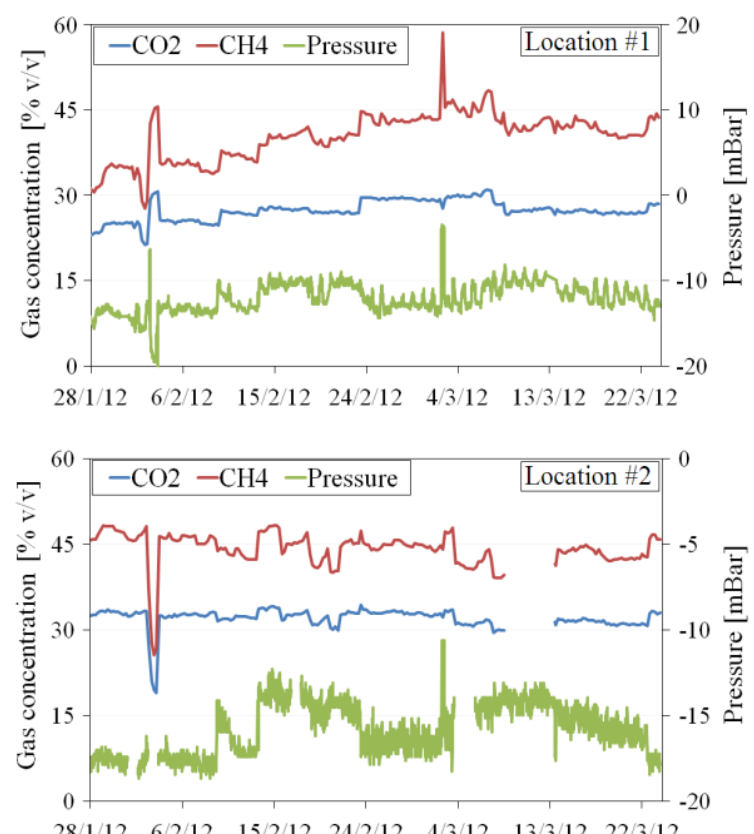

Figure 5: Subset of monitored data, where 'events' are evident in both gas and pressure readings

The variations in the constituent gas levels tended to be correlated, i.e. $\mathrm{CO}_{2}$ and $\mathrm{CH}_{4}$ tend to rise and fall at the same time. This indicated that the gas activity was predominantly controlled by a singular factor affecting the entire volume of the gas, most likely the extraction rate towards the flare. However, it can be seen that $\mathrm{CH}_{4}$ at times experienced a greater differential change possibly due to other factors, such as varying methanogenic activity of the waste body associated with the different stages of waste decomposition and increased moisture content after rainfall. Clearly, the availability of near real-time methane concentration variation across the site can assist in field-balancing to attain the optimum gas composition for combustion in the flare.

Pressure readings also tended to align with gas activity. This was an interesting finding: given their substantially lower cost, greater proportion of pressure sensors could be deployed for the same investment. A relationship established between pressure and gas would enable pressure sensors to be deployed as low-cost indicative monitors, signalling a possible change in gas concentration levels which could then be verified by a smaller number of gasspecific sensors. Judging from Figure 5, the correlation between gas and pressure appeared stronger for location \#1 compared to Location \#2; the investigation of the relationship between gas and pressure is subject to further careful consideration.
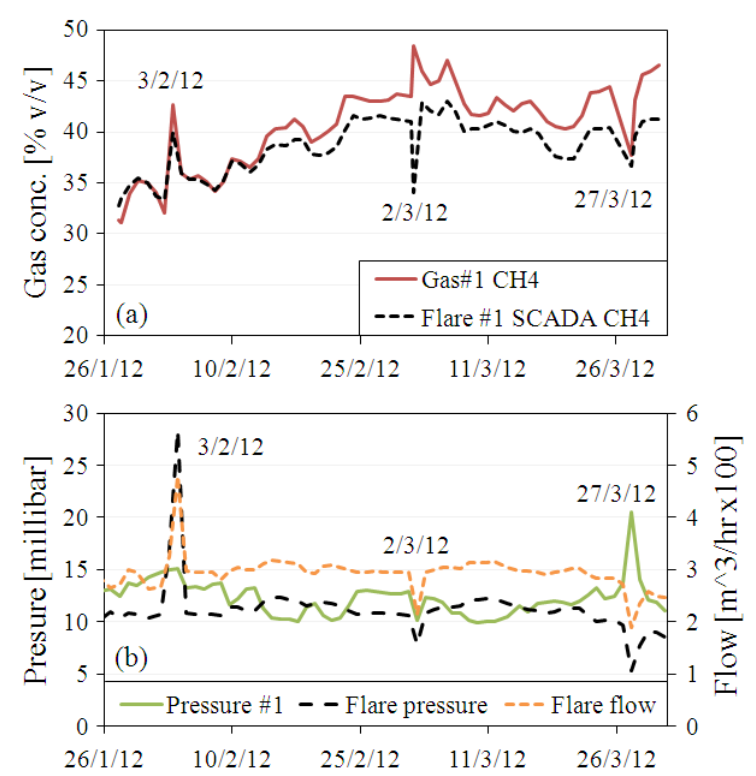

Figure 6: Subset of data indicating correlation between location \#1 deployed platforms and SCADA (a) gas comparison (b) pressure/flow comparison

Table 1: Correlation analysis of deployed systems and SCADA measurements (values approaching \pm 1.0 indicate stronger correlation).

\begin{tabular}{|c|c|c|c|c|c|}
\hline \multirow{2}{*}{$\begin{array}{l}\text { Deployed } \\
\text { platforms }\end{array}$} & \multicolumn{4}{|c|}{ SCADA } \\
\cline { 2 - 6 }$\# 1$ & $\mathrm{CH}_{4}$ & $\mathrm{CO}_{2}$ & Pressure & Flow \\
\hline \multirow{3}{*}{$\# 1$} & $\mathrm{CH}_{4}$ & 0.72 & - & -0.024 & -0.005 \\
\cline { 2 - 6 } & $\mathrm{CO}_{2}$ & - & 0.736 & 0.059 & 0.107 \\
\cline { 2 - 6 } & Pressure & - & - & 0.065 & 0.218 \\
\hline \multirow{3}{*}{$\# 2$} & $\mathrm{CH}_{4}$ & 0.649 & - & 0.195 & 0.358 \\
\cline { 2 - 6 } & $\mathrm{CO}_{2}$ & - & 0.592 & 0.337 & 0.505 \\
\cline { 2 - 6 } & Pressure & - & - & 0.376 & 0.307 \\
\hline
\end{tabular}


Correlation analysis was conducted between the platforms' data and the flares' SCADA data. The correlation for two months (64 data points) is demonstrated in Figure 6 with correlation coefficients presented in Table 1. Reasonably strong correlations for gas concentrations were found for both locations and their respective SCADA measurements. This serves to validate the gas data collected by the deployed platforms. Interestingly, this correlation is not consistently positive, e.g. negative correlations in gas levels on $2 / 3 / 12$ and in pressure on 27/3/12 as seen in Figure 6. Gas activity measured locally at a specific point in the gas field does not necessarily manifest at the flare. This underscores the incapability of the flare's SCADA measurements to fully describe behaviour in gas activity further along the extraction system - a task that could be accomplished by distributed sensor network.

The correlations in gas concentration levels were slightly higher for location \#1 compared to location \#2 (0.73 vs. 0.62 averaged); as this difference is slight, no major conclusions are being drawn though this is subject to further investigation. Conversely, correlations for pressure/flow were substantially stronger for location $\# 2 \quad(>0.3$ vs. $<0.2)$; the interrelationship was less evident for location \#1 possibly due to other gas inlets to that particular flare.

A lesser correlation is seen between the $\mathrm{CH}_{4}$ values and SCADA flowrate compared to that of $\mathrm{CO}_{2}$ (0 vs. 0.1 for location $\# 1 ; 0.35$ vs. 0.5 for location \#2). It is difficult to ascribe variations in a system as complex as a landfill gas field to a specific source; one possibility is differing levels of methanogenic activity in the waste body according to varying conditions of waste decomposition.

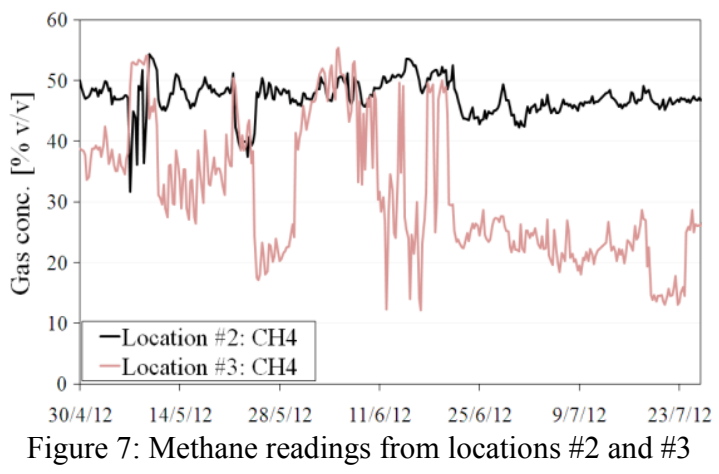

The simultaneous measurement of gas levels at different points in the landfill extraction system is another advantage provided by distributed sensor platforms. Location \#3 was situated on an extraction line leading to the flare via location \#2, see Figure 1(ii). A subset of the $\mathrm{CH}_{4}$ data from both locations is displayed in Figure 7. $\mathrm{CH}_{4}$ generation in location \#3 is seen to be less than that in Location \#2 - this is to be expected for a new-capped waste cell where waste decomposition is not at a sufficiently advanced stage. The elevated $\mathrm{CH}_{4}$ levels in location \#2 indicate that the field is balanced from other cells to provide the appropriate composition for the flare. Interestingly, $\mathrm{CH}_{4}$ levels in location \#3 matches those in location \#2 on a few occasions; the authors estimate that the extraction rate from location \#3 was increased, hence reducing the $\mathrm{CH}_{4}$ concentration by allowing less time for the gas to accumulate. This finding illustrates the benefit of immediate feedback following field-balancing, allowing for more precise and effective gas management.

\section{CONCLUSIONS}

Autonomous monitoring platforms have been developed for the application of monitoring gas on landfill sites. This paper describes the deployment of a wireless sensor network on an active landfill site, the first of its type in Ireland. The near real-time access to gas concentration and pressure data via an online portal enabled the landfill operators and EPA to characterise, for the first time, the dynamics of landfill gas activity. The authors aim to continue working closely with landfill operators, demonstrating that the integration of data arising from distributed sensor networks into landfill operational practice would assist in field-balancing and gas management. Furthermore, the cost-benefit of this service will be promoted where the operation of the engine/flare can be more effectively managed. At this approximately half-way point in the deployment duration, the preliminary analysis presented in this paper illustrates the valuable insight provided by knowledge of trends in landfill gas activity; more comprehensive analysis is ongoing with the deployment projected to continue until March 2013.

Further development of the platforms will involve implementing different communications protocols and reducing platform costs. The current use of GSM modules for all platforms is not financially viable when considering the extrapolated running costs associated with a greater magnitude of deployed systems. Reducing costs to a viable pricepoint is an integral part of the commercialisation process of this technology; in a sector that is becoming increasingly financially restricted, the 
ideal scenario of installing a sufficient number of platforms to fully encapsulate the gas field activity is currently too cost-prohibitive. Despite this, the authors believe that a viable commercial prospect exists with the increased temporal and spatial resolution provided by distributed monitoring platforms complemented with value-added analysis and interpretation of landfill gas data.

\section{ACKNOWLEDGEMENTS}

The authors wish to acknowledge funding from the EPA under the STRIVE programme (grant no. 2010ET-MS-10), and to thank the EPA and the landfill facility management for their co-operation

\section{REFERENCES}

European Parliament and Council of the EU, 2008. Directive 2008/50/EC on ambient air quality and cleaner air for Europe.

EPA, 2012. Ireland's Environment - An Assessment. Environmental Protection Agency, Ireland.

Cohen, R., Teige, V., Weichsel, K., 2012. The Berkeley Atmospheric $\mathrm{CO}_{2}$ Observation Network. URL: http://beacon.berkeley.edu/

Envirowatch, 2012. URL: http://www.envirowatch.ltd.uk

Beirne, S., Kiernan, B., Fay, C., Foley, C., Corcoran, B., Smeaton, A.F., Diamond, D., 2010. Autonomous greenhouse gas measurement system for analysis of gas migration on landfill sites. In: SAS 2010 - IEEE Sensors Applications Symposium, Limerick, Ireland.

Fay, C., Doherty, A., Beirne, S., Collins, F., Foley, C., Healy, J., Kiernan, B., Lee, H., Maher, D., Orpen, D., Phelan, T., Qiu, Z., Zhang, K., Gurrin, C., Corcoran, B., O'Connor, N., Smeaton, A.F., Diamond, D., 2011. Remote real-time monitoring of subsurface landfill gas migration, Sensors, vol. 11, pp. 6603-6628.

Collins, F., Orpen, D., Fay, C., Diamond, D., 2012. Analysis of landfill gas migration by use of autonomous gas monitoring platforms. Proceedings of The $27^{\text {th }}$ International Conference on Solid Waste Technology and Management, Philadelphia, USA.

Collins, F., Orpen, D., Fay, C., Foley, C., Smeaton, A.F., Diamond, D., 2011 Web-based monitoring of yearlength deployments of autonomous gas sensing platforms on landfill sites. Proceedings of IEEE SENSORS 2011, Limerick, Ireland. 